\title{
Lipopolysaccharide Binding Protein and Oxidative Stress in a Multiple Sclerosis Model
}

\author{
Begoña M. Escribano ${ }^{1,2}$ - Francisco J. Medina-Fernández ${ }^{2,3}$. \\ Macarena Aguilar-Luque ${ }^{2,3} \cdot$ Eduardo Agüera $^{2,4} \cdot$ Montserrat Feijoo $^{2,3}$. $^{2}$ \\ Fe I. Garcia-Maceira ${ }^{5}$ - Rafael Lillo ${ }^{2,6}$ - Patricia Vieyra-Reyes ${ }^{7}$ - Ana I. Giraldo ${ }^{2,3}$. \\ Evelio Luque $^{2,8}$ • René Drucker-Colín ${ }^{9}$ - Isaac Túnez ${ }^{2,3,10}$
}

Published online: 7 October 2016

(C) The American Society for Experimental NeuroTherapeutics, Inc. 2016 sults of this study revealed an improvement in the clinical symptoms of the EAE and MS with the treatments, as well as a reduction in the oxidative stress parameters and in LBP. Correlations between the clinical variables of the disease, i.e. oxidative damage and LBP, were established. Although the conclusions of this research are indeed relevant, further investigation would be necessary to establish the intrinsic mechanisms of the MS-oxidative stress-microbiota relationship.

Keywords Dimethyl fumarate $\cdot$ Experimental autoimmune encephalomyelitis $\cdot$ Lipopolysaccharide binding protein . Natalizumab · Oxidative stress $\cdot$ Relapsing-remitting multiple sclerosis

Begoña M. Escribano and Francisco J. Medina contributed equally to this work.

Electronic supplementary material The online version of this article (doi:10.1007/s13311-016-0480-0) contains supplementary material, which is available to authorized users.

Isaac Túnez

fm2tufii@uco.es

1 Departamento de Biologia Celular, Fisiologia e Inmunologia, Facultad de Veterinaria, Universidad de Cordoba, Cordoba, Spain

2 Instituto Maimonides de Investigacion Biomedica de Cordoba (IMIBIC), Cordoba, Spain

3 Departamento de Bioquimica y Biologia Molecular, Facultad de Medicina, Universidad de Cordoba, Cordoba, Spain

4 Servicio de Neurología, Hospital Universitario Reina Sofía de Cordoba, Cordoba, Spain

5 Canvax Biotech SL, Parque Científico Rabanales 21, Cordoba, Spain

6 Departamento de Ciencias Sociosanitarias y Radiologia y Medicina Fisica, Seccion de Psiquiatria, Facultad de Medicina, Universidad de Cordoba, Cordoba, Spain

7 Departamento Neurofisiología de la Conducta, Facultad de Medicina, Universidad Autonoma del Estado de México, Toluca, Estado de Mexico, Mexico

8 Departamento de Ciencias Morfologicas, Seccion Histologia, Facultad de Medicina, Universidad de Cordoba, Cordoba, Spain

9 Departamento de Neuropatologia Molecular, Instituto de Fisiologia Celular, Universidad Nacional Autonoma de Mexico (UNAM), Ciudad de Mexico, D.F., Mexico

10 Red Tematica de Investigacion Cooperativa en Envejecimiento y Fragilidad (RETICEF), Cordoba, Spain 


\section{Introduction}

Multiple sclerosis (MS) is a chronic, disabling disease of the central nervous system (CNS) that has an autoimmune component. This disease is characterized by chronic neuroinflammation with lymphocyte infiltration into the CNS, myelin loss, gliosis, various degrees of axonal and oligodendrocyte pathology and progressive neurological dysfunction [1, 2]. Moreover, MS is a disease with profound heterogeneity in its clinical course, a neuroradiologic appearance in its lesions, the involvement of susceptibility gene loci, and different responses to therapy [3]. Recent studies have begun to elucidate the contribution of microbiome and its relevant factors to MS pathogenesis, with much of the work being investigated in experimental encephalomyelitis (EAE) models $[4,5]$. EAE is a widely used animal model of MS induced by CNSrestrictive antigens [5]. For this study, the EAE inductor agent chosen was myelin oligodendrocyte glycoprotein (MOG). MOG induction shows several similarities to MS, such as a relapsing-remitting (RR) disease course, demyelination, and axonal degeneration $[2,6]$.

Microbes colonize all the surfaces of the human body exposed to the environment, with the majority residing in the intestinal tract [7]. Evidence supporting a role of intestinal microbiota in triggering brain autoimmunity stems from EAE models. One classical approach has relied on purging the gut flora using antibiotics. In this context, it has been reported that antibiotic treatment directed towards altering gut microflora suppresses EAE [8, 9].

Direct proof identifying the commensal gut flora as prompting brain autoimmunity came from the RR mouse model. RR mice housed in clean, specific pathogen-free conditions develop EAE typically within 3 to 8 months of age. When transferred to germ-free conditions, however, the mice remain disease-free. This is not due to a weakened immune system unable to mount a proper autoimmune attack, because after recolonization with commensal gut microbiota, the recipients promptly respond with typical EAE [9]. Legroux and Arbour [10] showed that lipopolysaccharide (LPS) binding protein (LBP), as a biomarker of gut microbiota, could be essential in triggering spontaneous brain autoimmunity in mice, and the abundance of immune cells such as $\mathrm{T}$ lymphocytes and their products in CNS lesions of patients with MS supports the notion that MS is an immune-mediated disorder.

However, no study relating gut microbial, LPS or LBP signatures to the development and progression of MS has been published, although recent findings in EAE suggest that altering certain bacterial populations present in the gut may lead to a proinflammatory condition that could result in the development of autoimmune diseases, in particular MS [4, 11]. In fact, 2 recent studies have demonstrated a gut microbial dysbiosis in patients with MS, although both of them have posited that further research would be necessary to establish the role of microbiota in the etiopathogenesis of MS $[12,13]$.

Compositional changes in microbiota can be indirectly reflected by the metabolites and co-metabolites of microbiota [5]. Certain researchers have provided extensive evidence regarding the induction of regulatory response induced by bacterial polysaccharides $[4,14,15]$. A bacterial LPS leads to the dysfunction of the mucosal barrier and affects other tissues when its plasmatic levels increase [16]. In dysbiotic microbiota, the gut endotoxin/LPS is increased, regulatory T cells are defective, and the aryl hydrocarbon receptors and proinflammatory $\mathrm{T}$ helper (Th) 17 cells are activated [17-19].

Moreover, LPS regulates the expression of inflammatory proteins associated with inflammatory diseases. Many studies have also shown that reactive oxygen species (ROS) are the major signaling molecules that mediate microglial activation induced by inflammatory mediators, including LPS [20].

ROS/reactive nitrogen species have a relevant function in MS pathogenesis [21-25]. Recent work has shown that gut epithelia contacted by enteric commensal bacteria rapidly generate ROS. Additionally, ROS have been seen to serve as critical second messengers in multiple signal transduction pathways stimulated by proinflammatory cytokines and growth factors [26]. Patients with MS have elevated levels of oxidative stress biomarkers, together with a global antioxidant deficiency [21]. The development of an effective antioxidative therapy for patients with MS is complex owing to the heterogeneity of the pathophysiologic mechanisms involved [27].

With this background, we have addressed the ideas that: 1) the changes in LPS and LBP are related to the development and evolution of MS and EAE; and 2) antioxidant therapies may improve or block the development of neurodegenerative diseases, specifically MS, at least partly, through their effects on LBP.

\section{Methods}

\section{Animals}

Animals from the Animal Experimentation Center (Cordoba University, Cordoba, Spain) were housed under standard colony conditions: $12 \mathrm{~h}: 12 \mathrm{~h}$ light/darkness cycle (lights on at 7:00 a.m.), controlled room temperature $\left(22 \pm 2{ }^{\circ} \mathrm{C}\right)$, with free access to food and water. This study was carried out in strict accordance with the recommendations in the Guide for the Care and Use of Laboratory Animals of the National Institute for Health. The protocols were approved by the Bioethics Committee at Cordoba University and carried out according to the guidelines of the Directive of 24 November 1986 (86/609/ECC) approved by the European Communities 
Council and RD 53/2013 approved by Presidency Minister of Spain (BOE 8 February 2013).

\section{Procedure}

\section{EAE Course Evolution with Sex}

A total of 29 Dark Agouti rats (2 months old, weighing 190-220 g) were used as this strains bears the closest clinical and pathologic resemblance to MS [28]. The rats were divided into 3 groups, 2 control groups, and 1 EAEinduced group. The first group, called "control", was not manipulated ( $n=10 ; 5$ males, 5 females), the second group ("sham") was inoculated with complete Freund's adjuvant ( $n=10 ; 5$ males, 5 females), and the third group, "EAE", was disease-induced with MOG ( $n=9 ; 5$ males, 4 females), the lower number in this group being attributed to the death of 1 animal from natural causes once the procedure had started.

Induction of EAE was performed by injecting subcutaneously, at the dorsal base of the tail, $100 \mu \mathrm{l}$ of a solution containing $150 \mu \mathrm{g}$ MOG (fragment 35-55; Sigma-Aldrich, Madrid, Spain) in phosphate-buffered saline emulsified 1:1 in complete Freund's adjuvant (Sigma-Aldrich, St. Louis, MO, USA). To complete the adjuvant, $400 \mu \mathrm{g}$ heatinactivated Mycobacterium tuberculosis (H37Ra; DIFCO, Detroit, MI, USA) was added. Sham induction was performed in control group (sham) animals by subcutaneous injection of $100 \mu \mathrm{l}$ complete Freund's adjuvant.

In this test, the clinical score agreement on a severity scale and EAE clinical signs were determined at the end of the experimental period of 35 days.

\section{Clinical and Oxidative Stress, LPS and LBP in EAE}

Five male rats were used for each of the following groups: 1) Control; 2) sham; 3) EAE; 4) EAE + natalizumab (EAE + nata); $\mathrm{EAE}+$ dimethyl fumarate $(\mathrm{EAE}+\mathrm{DMF}) ; \mathrm{EAE}+N-$ acetylcysteine (EAE + NAC).

Control, sham, and EAE rats were induced as was explained in the first test. The application pattern of the different drugs was as follows.

Based on the dose of $300 \mathrm{mg}$ delivered to patients with MS once every 4 weeks (life equivalency and physiological features in humans and rat), natalizumab (Tysabris; Biogen Idec, Inc.; and Elan Pharmaceuticals, Inc. Cambridge, MA, USA) was administered intraperitoneally at doses of $5 \mathrm{mg} / \mathrm{kg}$ weight every 10 days for 21 days, which means that the animal received 2 doses of natalizumab [29,30].

NAC administration (CINFA S.A., Pamplona, Spain) was performed at a dose of $1.4 \mathrm{~g} / \mathrm{kg}$ weight, oral administration, in drinking water and was administered daily for 21 days [31].
Oral intake makes DMF be rapidly metabolized into methyl fumarate, which is a bioactive metabolite. The dose was $15 \mathrm{mg} / \mathrm{kg}$ weight, oral administration, for a 21-day period [32].

In this test, the clinical signs, score agreement on a severity scale, oxidative damage markers, and LBP for each group were determined. LPS was also established but only in the control, sham, and EAE groups. See Supplementary material $\mathrm{S} 1$ for further information.

\section{Neurohistologic Changes and Apoptosis}

Neurohistologic changes were determined in 5 animals for each group indicated previously (treatment and EAE) by counting their neuronal nuclei and brain astrocytes. Moreover, lactate dehydrogenase (LDH) and caspase-3 levels were analyzed as apoptosis signs, in blood and brain tissues.

\section{Patients with RRMS Treated with Natalizumab}

Following acquisition of written informed consent, 6 healthy controls and 8 patients with RRMS ( 2 men, 6 women) were recruited from the Department of Neurology at Queen Sofia University Hospital (Cordoba, Spain). The revised McDonald criteria [33] were used and the patients were treated with $300 \mathrm{mg}$ natalizumab administered intravenously every 4 weeks (28 days) in concordance with current Spanish guidelines during 56 weeks (MS-56) (Table 1). The natalizumab was injected between 16:00 and 18:00 h.

Blood samples were taken from the antecubital vein immediately prior to the first natalizumab infusion (baseline) and after the fourteenth infusion (MS-56) of treatment. Samples were placed in chilled tubes without any anticoagulant, or with $1 \mathrm{mg} / \mathrm{ml}$ ethylenediaminetetraacetic acid (EDTA)-K3 (Becton-Dickinson, Franklin Lakes, NJ, USA) as an anticoagulant. Blood was collected

Table 1 Characteristics of patients with relapsing-remitting multiple sclerosis (RRMS)

\begin{tabular}{ll}
\hline Patients (male/female), $n$ & $2 / 6$ \\
Age (years) & $41 \pm 7.8(32-51)$ \\
EDSS & \\
$\quad$ Baseline & $4.1 \pm 1.6$ \\
$\quad$ MS-56 & $4.1 \pm 1.4$ \\
Disease duration (years) & $6.1 \pm 2.6(3-10)$ \\
Relapses before treatment $(n)$ & $4.5 \pm 1.5$ \\
Relapses during treatment $(n)$ & $0.5 \pm 0.7^{\mathrm{a}}$ \\
\hline
\end{tabular}

Values are expressed as mean $\pm \mathrm{SE}$ unless otherwise indicated. Baseline: patients with MS before treatment (baseline) and after 56 weeks of treatment with natalizumab (MS-56). EDSS = Expanded Disability Status Scale

${ }^{\mathrm{a}} p<0.05$ vs baseline group 
between 15:30 and 17:30 h. Plasma was separated from red blood cells by centrifugation at $1600 \mathrm{~g}$ at $4{ }^{\circ} \mathrm{C}$ for $15 \mathrm{~min}$. Aliquots of supernatant $(0.5-1 \mathrm{ml})$ were immediately stored at $-85^{\circ} \mathrm{C}$ until analysis. All oxidative stress biomarkers were measured at the Department of Biochemistry and Molecular Biology, Faculty of Medicine, University of Cordoba. Laboratory staff were blinded to LBP and LPS biomarkers at all stages of analysis. The study was carried out in accordance with the Declaration of Helsinki and was approved by the local ethical committee of Queen Sofia University Hospital, Spain.

\section{Sample Collection and Processing}

At 35 days, the animals were killed by decapitation having been previously anesthetized with an intraperitoneal injection of ketamine $75 \mathrm{mg} / \mathrm{kg}$ (Imalgene $100 \mathrm{mg} / \mathrm{ml}$; Merial Laboratorios, Barcelona, Spain). The blood obtained from neck vascular trunk was collected in tubes with EDTA-K3. Tubes were centrifuged for $15 \mathrm{~min}$ at $1900 \mathrm{~g}$ at $4{ }^{\circ} \mathrm{C}$, proceeding immediately to the collection of plasma and storage in aliquots at $-85^{\circ} \mathrm{C}$.

Then, and under controlled temperature conditions, the brain and spinal cord were extracted and weighed, and the corresponding homogenates immediately prepared with a mechanical homogenizer (Tempest Virtis, Labsquip, Markham, Canada). The buffer used for homogenization was Tris $(20 \mathrm{mM})$ at $\mathrm{pH} 7.4$.

For the histologic study, whole brains were rapidly removed and fixed by immersion in $10 \%$ buffered formaldehyde. Subsequently, they were embedded in paraffin wax, cut into eight $\mu \mathrm{m}$-thick sections and stained with $0.025 \%$ cresyl violet (Nissl-stained). For the survey of the number of astrocytes, the brains were dehydrated with increasing solutions of sucrose (10-30\%) after binding and immersion in $4 \%$ of buffered formaldehyde. They were then embedded in paraffin wax, cut into eight $\mu \mathrm{m}$ thick sections.

\section{Variables Analyzed}

The following parameters were analyzed.

\section{Clinical Score Evaluation}

The animals were monitored at 14 and 35 days and scored in accordance with the following severity scale: $0=$ no signs; $1=$ tail paralysis; $2=$ weakness in hindlimbs; $3=$ paralysis in hindlimbs; $4=$ paralysis in hindlimbs and weakness in forelimbs; $5=$ quadriplegic [2]. The increase between score and disease was established (score at 35 days - score at 14 days). Other symptoms were observed, such as inflammation (edema appearance) and fur status. The patients with RRMS were examined clinically using the Expanded Disability Status Scale [34].

\section{Oxidative Damage Biomarkers in Brain and Spinal Cord}

Lipid peroxides (LPO; nmol/mg protein), total glutathione (tG; nmol/mg protein), reduced glutathione (GSH; nmol/mg protein), oxidized glutathione (GSSG; $\mathrm{nmol} / \mathrm{mg}$ protein), and the quotient between them (ratio GSH/GSSG) were all analyzed by spectrophotometry with Bioxytech S.A. reagents (Oxis International, Portland, OR, USA) at the Department of Biochemical and Molecular Biology of the Medicine Faculty at Cordoba University. All samples were analyzed with a Shimadzu spectrophotometer (UV 1603; Shimadzu, Kyoto, Japan) and the reagent kits were LPO 586 (LPO), GSH 420 (tG), GSH 400 (GSH), and GSH 412 (GSSG).

In blood, carbonylated proteins were measured $(\mathrm{nmol} / \mathrm{g}$ hemoglobin) as an indicator of protein oxidative stress. The carbonyl content was evaluated using the method of Levine et al. [35]. The evaluation was made in a Shimadzu spectrophotometer (UV-1603) at a wavelength of $360 \mathrm{~nm}$.

\section{LDH in Blood and Brain Tissues}

LDH catalyzed the reaction of pyruvate in the presence of nicotinamide adenine dinucleotide to lactate. The diminution speed of nicotinamide adenine dinucleotide concentration in the photometrically examined medium was proportional to the concentration catalytic LDH in the tested sample. Determination was performed spectrophotometrically with Labkit reagents in a Shimadzu spectrophotometer (UV-1603).

\section{Caspase-3 Determination in Brain and Blood}

The reagents employed were Caspase-3/CPP32 Colorimetric Assay Kit (BioVision, Milpitas, CA, USA). This assay was based on spectrophotometric detection of the Chromophore $P$-nitroaniline ( $p \mathrm{NA}$ ) after cleavage from the labeled substrate N-Acetyl-Asp-Glu-Val Asp $p$ nitroaniline (DEVD- $p \mathrm{NA}$ ). The $p \mathrm{NA}$ light emission can be quantified using a spectrophotometer at 400 or $405 \mathrm{~nm}$ (Shimadzu UV-1603). Comparison of the absorbance of $p$ NA from an apoptotic sample to an uninduced control allows the determination of the fold increase in CPP32 activity.

\section{LPS in Spinal Cord and Blood, Both in EAE Rats and in Blood of Patients with RRMS}

LPS was assessed using the Pierce (\#61666) LAL Chromogenic endotoxin quantification kit provided by Thermo Scientific (Waltham, MA, USA). LPS catalyzes the 
activation of a proenzyme in the modified Limulus amebocyte lysate assay, which then catalyzes the splitting of $p$ NA. The activation rate is proportional to the sample endotoxin concentration. The released $p \mathrm{NA}$ is photometrically measured at 405-410 nm. Data are expressed as endotoxin units/mg protein or mg hemoglobin.

\section{LBP in Brain Tissue, Spinal Cord, and Blood}

LBP is upregulated as part of the acute phase response and serves as a marker of endotoxemia by bacterial lipopolysaccharides (components of the outer membrane of Gramnegative bacteria capable of inducing strong immune responses). In addition, LBP levels might indirectly translate variations/alterations as occurrs in gut microbiota. LBP assessment was performed using Elisa Kit soluble LBP (Enzo Life Sciences, New York, NY, USA) for humans or rats. The amount of LBP was measured in an enzyme-linked immunosorbent assay reader. The values are presented as $\mathrm{pg} / \mathrm{mg}$ protein or $\mathrm{pg} / \mathrm{g}$ hemoglobin.

\section{Tissue Processing and Histologic Analysis}

Ten random areas were examined at stratium core level, in $40 \times$ zoom leading to an area of $35,500 \mu^{2}$. The number of cores was quantified semiautomatically with the Image Pro Plus program (Media Cybernetics, Cambridge, UK).

The number of astrocytes was determined by immunohistochemistry. The antibodies used were antiglial fibrillary acidic protein (Abcam, Cambridge, UK) as the primary one and donkey antigoat Alexa 555 (Invitrogen, Carlsbad, CA, USA) as the secondary one. With a Leica fluorescence microscope (dM2500) and an image overlay program, Image 1.49, and the Image Pro Plus counting program, nuclei and astrocytes were counted in 10 random areas with $40 \times$ zoom, each area being $35,500 \mu^{2}$. For the counting, the red images (astrocytes) overlaid the blue ones (nuclei). The core and astrocyte accumulation was tabulated as 1 .

\section{Statistics}

The statistical study was performed with the SPSS (version 15 for Windows; IBM, Armonk, NY, USA). First, the different quantitative variables understood in the study were returned to normality by using the Shapiro-Wilk test for $n<40$. Once proven that the values had returned to a normal distribution, a Student's $t$ test for independent variables and one for paired data for dependent variables were conducted in the first trial. For the second and third trials, a hypothesis contrast of each of the variables in the different groups established was performed by applying a 1-way analysis of variance. To determine the concrete differences between the groups, a Bonferroni test was carried out. A Pearson correlation between the variables LPO, clinical score at 35 days, and LBP in blood was carried out. For the fourth trial, 1-way analysis of variance and post hoc Bonferroni tests were conducted to compare the control to the baseline levels (prior to infusion) and the MS-56 (56 weeks postinfusion).

The minimum significance level was $95 \%(p<0.05)$. The results are expressed as arithmetic mean $\pm \mathrm{SE}$.

\section{Results}

\section{EAE Course Evolution with Sex}

The results show that were no differences in the course of the EAE for the clinical score and the oxidative damage markers between male and female rats (Supplementary material S2 and S3).

The animals inoculated with MOG, regardless of sex, had developed EAE (100\% of animals) at the end of the experiment at 35 days postinduction. The clinical score at 14 days, 35 days, and the increase in score between 14 and 35 days are shown in Supplementary material S2 for the different treatments (control; sham; EAE). All scores were significantly different from those obtained by the control group with $100 \%$ of healthy animals.

As biomarkers of oxidative stress in brain, LPO, tG, GSH, GSSG, and GSH/GSSG are shown in Supplementary material $\mathrm{S} 3$. There was an increase of LPO in EAE with respect to the control in the general group and in the males, and a decrease in the GSH/GSSG ratio in the control for the general group and the females. Once the first trial was completed, there were no differences in the course of the EAE for the oxidative damage markers between male and female rats. The second and third trials used only males, to prevent any interference from the hormones responsible for the females' estrus cycles.

\section{Clinical Conditions, Oxidative Stress, LPS, and LBP in EAE}

The results of the EAE clinical condition are shown in Fig. 1a, in which the different treatments (control; sham; EAE; EAE + nata; EAE + DMF; EAE + NAC) with their score at 14 and 35 days postinduction and the increase between both scores are given. In Fig. 1b, the score from 14 to 35 days every 3 days was monitored. All treatments manifested differences, with control and sham groups having $100 \%$ of healthy animals. $\mathrm{EAE}+\mathrm{NAC}$ treatment score showed a reduction to 35 days with respect to the EAE group, and an increased negative (1435 days) and different EAE group.

As biomarkers of oxidative stress in brain (Fig. 2a), there was a significant rise in all experimental groups compared with the control and sham groups in LPO. However, LPO was significantly reduced in the $\mathrm{EAE}+$ nata and $\mathrm{EAE}+$ NAC groups compared with EAE. There were no significant 
Fig. 1 a Clinical score: mean \pm SE of the clinical monitoring of the animals at 14 and 35 days, which was scored in accordance with the following increasing severity scale: $0=$ no signs; $1=$ tail paralysis; $2=$ hindlimb paralysis; $3=$ hindlimb partial paralysis; 4 = paralysis of hindlimbs with weakness in front ones; 5 = quadriplegic. In addition, the increase in score with the disease was established (score at 35 days - score at 14 days: score increment). ${ }^{* * * * *} p<0.001$ vs control; ${ }^{* * *} p<0.01$ vs control; ${ }^{*} p<0.05$ vs control; $\bullet \bullet p<0.001$ vs sham; $\bullet \bullet<0.01$ vs sham; $\bullet p<0.05$ vs sham; ${ }^{\# \#} p<0.01$ vs experimental autoinmune encephalitis (EAE); ${ }^{\#} p<0.05 v s$ EAE. Nata $=$ natalizumab; DMF = dimethyl fumarate; $\mathrm{NAC}=N$ acetylcysteine. b Clinical score: mean \pm SE of the clinical monitoring of the animals from 14 to 35 days every 3 days, which was scored in accordance with the severity scale described for (a). All this was carried out for the different treatment groups: control, sham, EAE, EAE + nata, $\mathrm{EAE}+\mathrm{DMF}$, and EAE+ NAC a
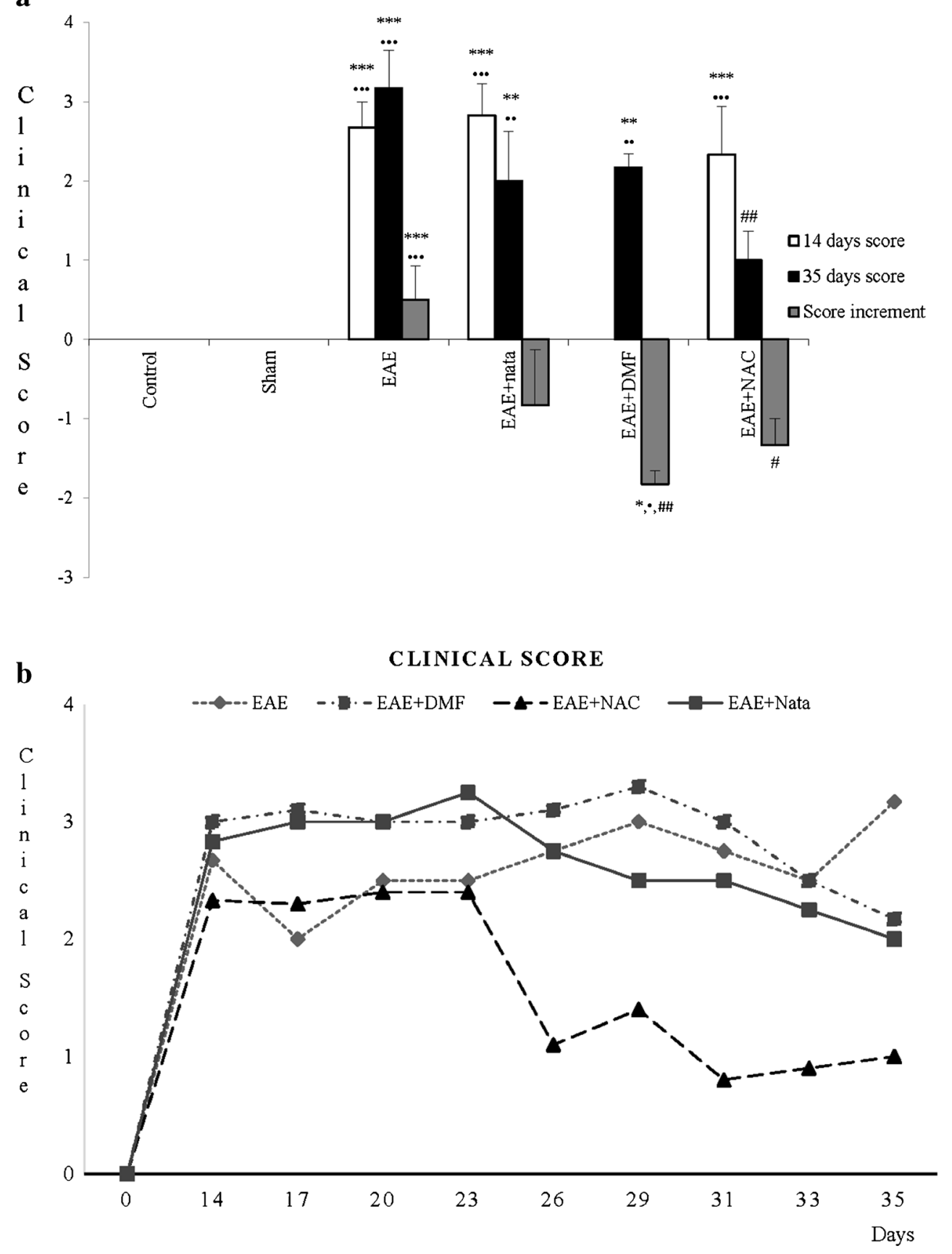

differences in the GSH/GSSG ratio between groups, except for $\mathrm{EAE}$ and $\mathrm{EAE}+\mathrm{NAC}$, with the latter showing a slightly higher average value than the former. However, LPO levels were significantly higher for all groups compared with the control in spinal cord (Fig. 2b), though the average LPO values in the treatment groups significantly decreased compared with the EAE group. There were no significant differences between groups for the GSH/GSSG ratio. Additionally, in blood (Fig. 2c), the carbonylated proteins were significantly higher in the EAE group. Treatment with natalizumab reduced levels of carbonylated proteins and matched those obtained from control and sham.

LBP in rat brain (Fig. 2a) and spinal cord (Fig. 2b) was significantly increased in the EAE group with respect to the other groups. Treatment with natalizumab, NAC, and DMF reduced LBP values to those present in control and sham. In blood (Fig. 2c), LBP was significantly higher in the EAE group. In clinical assays, treatment with natalizumab reduced levels of LBP and matched those from the control and sham groups. The correlations established were significant and 

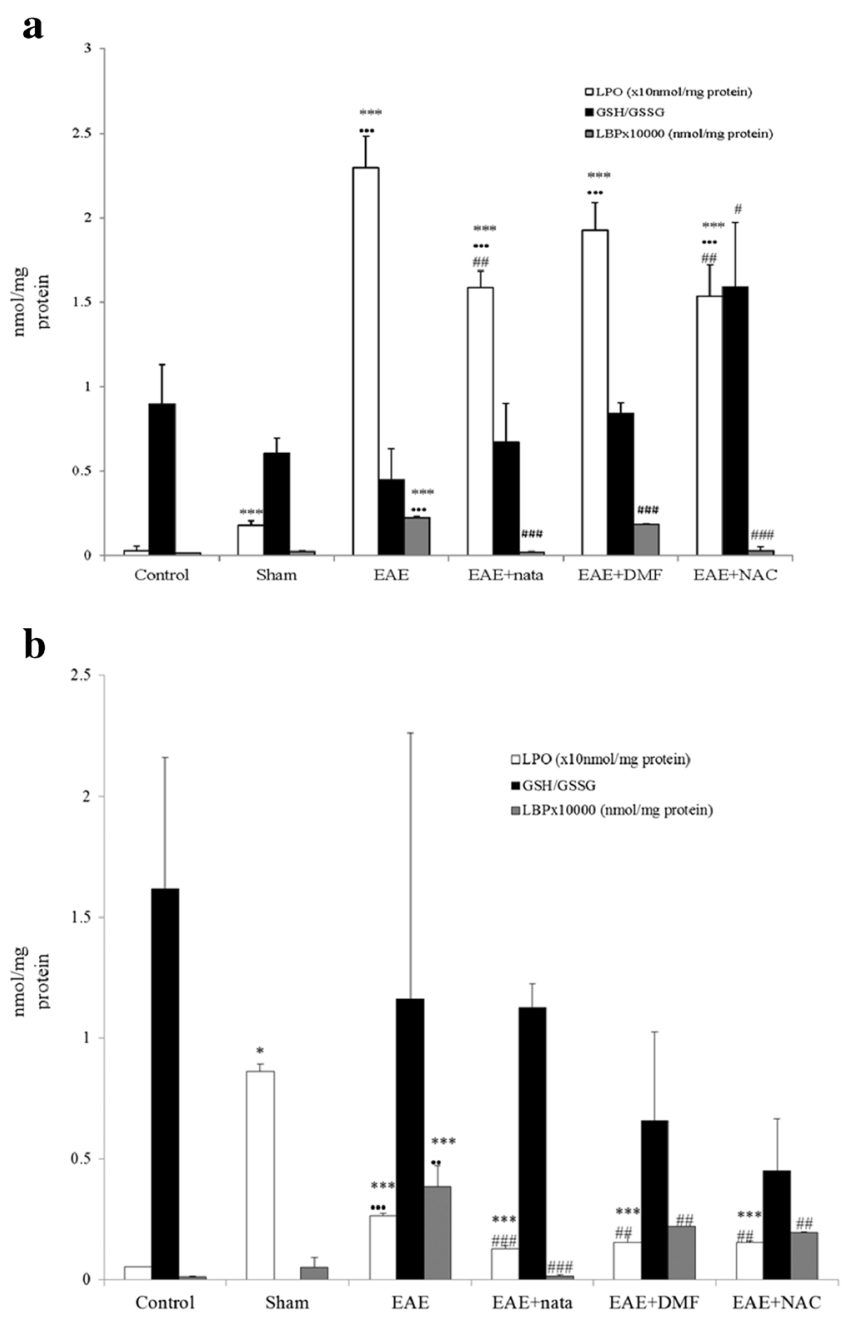

Fig. 2 a Brain: mean $\pm \mathrm{SE}$ in brain for the lipid peroxides (LPO; $\mathrm{nmol} / \mathrm{mg}$ protein), reduced glutathione quotient (GSH; nmol/mg protein)/oxidized glutathione (GSSG $\mathrm{nmol} / \mathrm{mg}$ protein), and the bacterial lipolysaccharide binding protein $(\mathrm{LBP} \times 10,000 \mathrm{pg} / \mathrm{mg}$ protein). b Spinal cord: mean $\pm \mathrm{SE}$ in the spinal cord for LPO (nmol/mg protein), GSH (nmol/mg protein)/GSSG (nmol/mg protein), and bacterial LBP $(\times 10,000 \mathrm{pg} / \mathrm{mg}$ protein). $\mathrm{c}$ Blood: mean $\pm \mathrm{SE}$ in blood for the carbonyl proteins (nmol/g hemoglobin), and bacterial LBP

positive for brain and spinal cord between puncture at 35 days and LPO [brain: $\mathrm{r}=0.786(p<0.001)$; spinal cord: $\mathrm{r}=0.736$ $(p<0.001)$ ] and LBP [brain: $\mathrm{r}=0.661(p<0.001)$; spinal cord: $\mathrm{r}=0.452(p<0.035)]$, also between increased rate 14-35 days and LBP $(\mathrm{r}=0.496 ; p<0.009)$ in brain and between LPO and LBP in brain and spinal cord [brain: $\mathrm{r}=0.630(p<0.001)$; spinal cord: $\mathrm{r}=0.668(p<0.002)]$. There was a negative correlation between $\mathrm{GSH} / \mathrm{GSSG}$ ratio in the brain and score at 35 days $(\mathrm{r}=-0.377 ; p<0.031)$.

In blood, there was a significant positive correlation $(\mathrm{r}=0.759 ; p<0.001)$ between carbonylated proteins and LBP levels.

LPS in rat spinal cord (Fig. 3a) and blood (Fig. 3b) was significantly increased in the EAE group with respect to the control and sham groups.

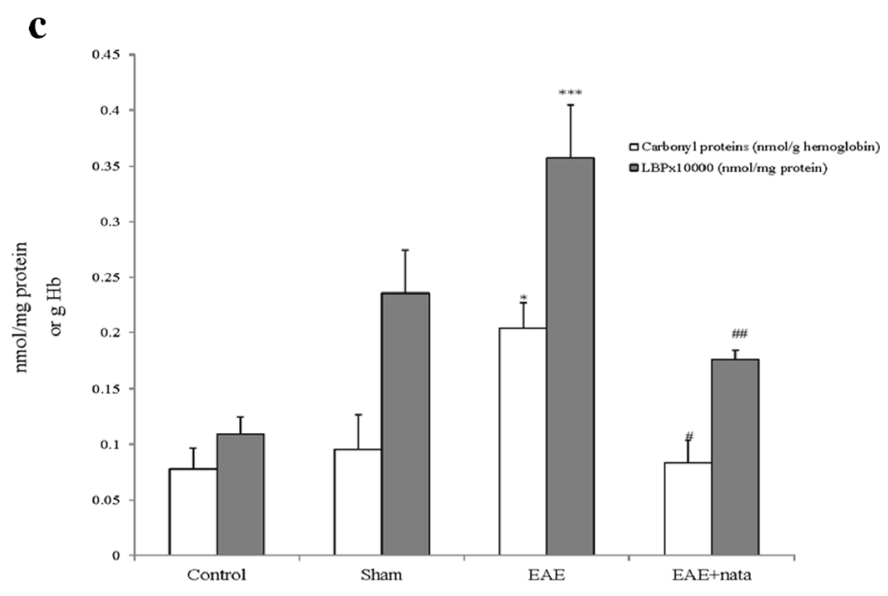

$(\times 10,000 \mathrm{pg} / \mathrm{mg}$ protein). $\mathrm{nmol} / \mathrm{g} \mathrm{Hb}=\mathrm{nmol} / \mathrm{g}$ hemoglobin. All this was carried out for the different treatment groups: control, sham, experimental autoimmune encephalitis (EAE), EAE + natalizumab (EAE + nata), EAE + dimethyl fumarate (EAE + DMF), and EAE + $N$-acetylcysteine (EAE + NAC). $* * * p<0.001$ vs control; $* p<0.05$ vs control; $\cdots p<0.001$ vs sham; $\bullet p<0.01$ vs sham; ${ }^{\# \#} p<0.001$ vs EAE; ${ }^{\# \#} p<0.01$ vs EAE; ${ }^{\#} p<0.05$ vs EAE

In conclusion, the results showed that the clinical score, oxidative stress, LPS, and LBP were increased in the EAE group with respect to control and sham groups. The different treatments reduced the clinical score, oxidative stress, and LBP with respect to the EAE group. Significant positive correlations were established between oxidative stress and LBP levels in the brain, spinal cord, and blood.

\section{Neurohistologic Changes and Apoptosis}

In our study, dead and damaged cell biomarkers underwent changes. Thus, LDH in the brain was significantly higher for the diseased animals relative to the vehicle and different treatments (Fig. 4a). 

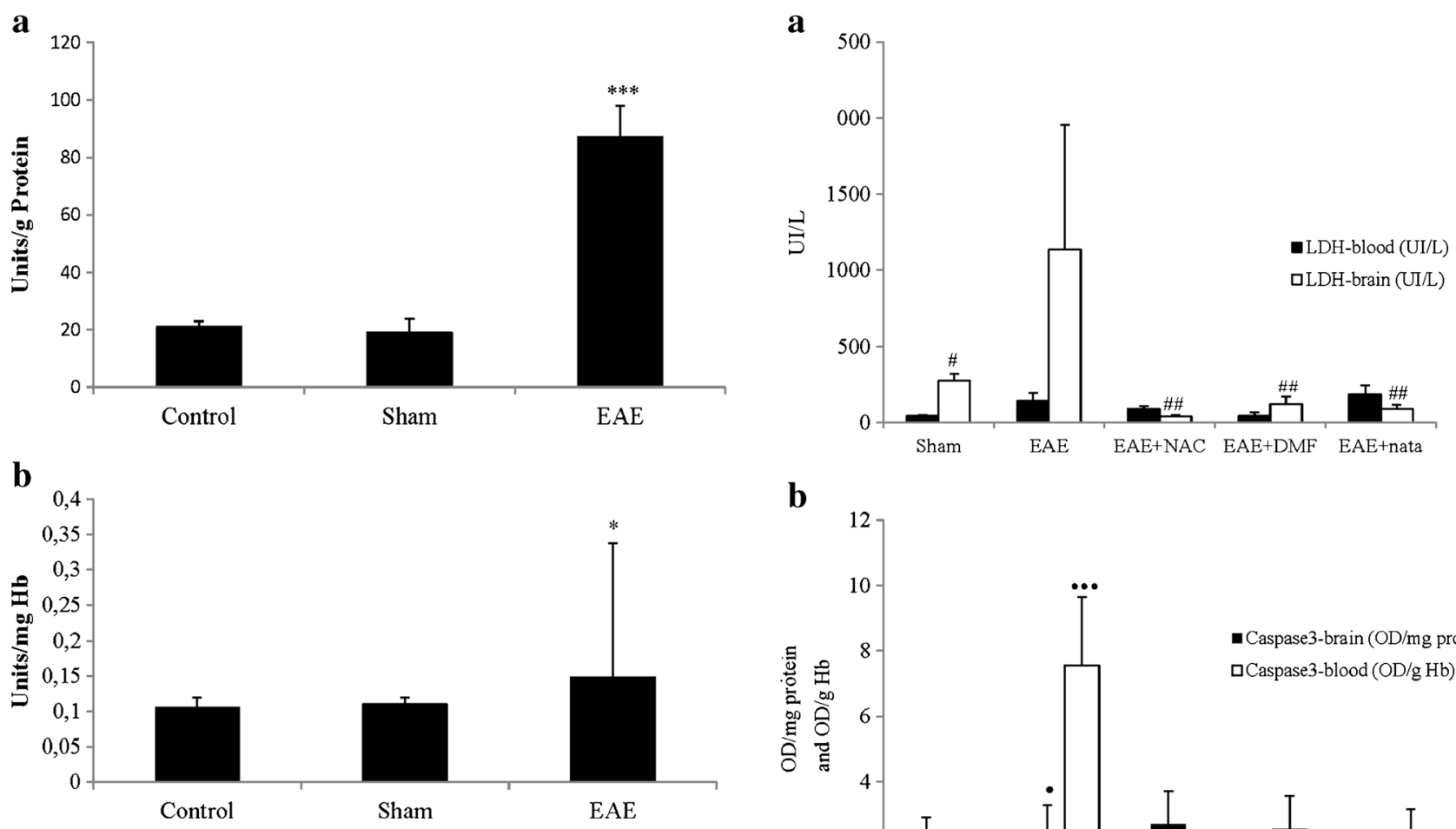

b

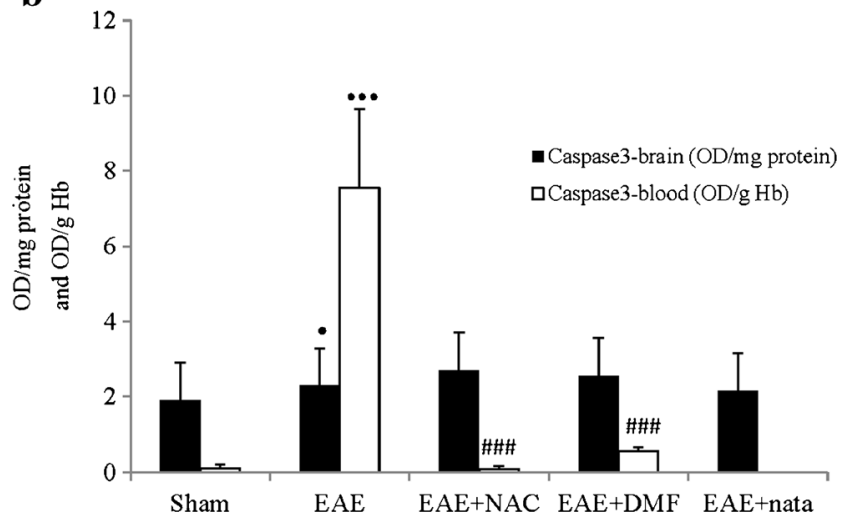

Fig. 4 a Lactate dehydrogenase (LDH) levels in brain and blood: Mean \pm SE for LDH levels in brain (UI/L) and blood (UI/L). b Caspase-3 in brain and in blood: mean $\pm \mathrm{SE}$ for Caspase- 3 in brain [optical density $(\mathrm{OD}) / \mathrm{mg}$ protein] and in blood [OD/g hemoglobin $(\mathrm{Hb})]$. All this was carried out for the different treatment groups: sham, experimental autoimmune encephalitis (EAE), EAE + natalizumab (EAE + nata), $\mathrm{EAE}+$ dimethyl fumarate $(\mathrm{EAE}+\mathrm{DMF})$, and $\mathrm{EAE}+N$-acetylcysteine

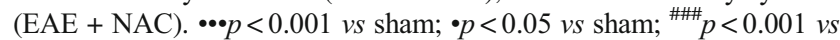
$\mathrm{EAE} ;{ }^{\# \#} p<0.01$ vs EAE; $\# p<0.05$ vs $\mathrm{EAE}$ with natalizumab and DMF reduced mean values of caspase- 3 in the blood relative to the EAE group.

The neuronal density in EAE significantly decreased compared with the control, and the number of astrocytes increased (Fig. 5a, b). Treatment with NAC increased neuronal density and significantly decreased the number of astrocytes.

\section{Patients with RRMS Treated with Natalizumab}

The number of relapses was also significantly reduced with natalizumab treatment at 56 weeks in patients with RRMS (Table 1).

Pretreatment LBP values in patients with RRMS were higher than in controls. At 56 weeks of treatment with natalizumab, these mean values were significantly reduced with respect to those at the start of the treatment (Fig. 6).

\section{Discussion}

MS is a chronic CNS demyelinating disease mediated by autoreactive immune attack against central neural tissues.

Dysregulation of the intestinal microbiota has been related to the course of MS, but its mechanism has not been established. However, the commensal gut flora - in the absence of pathogenic agents - is essential in provoking immune processes, leading to a relapsing-remitting autoimmune disease driven by myelin-specific $\mathrm{CD}^{+} \mathrm{T}$ cells [9]. However, oxidative stress, with a relevant function in MS, could be implicated. In this study, three of the treatments most used against MS were evaluated in order to investigate, at least partly, the relationship of MS microbiota and oxidative stress through their effects on LBP, as markers of changes in both LPS and gut microbiota.

No sex differences were reported in the first trial of this study. However, although in the experimental MS model sex differences in EAE susceptibility were initially described in rats, a great variability was observed between laboratories or within experiments [36]. 
Fig. 5 a Nuclei in the striated nucleus: mean \pm SE of nuclei in the striated nucleus in 10 random areas at $40 \times$, each area $=35,500 \mu^{2}$. b Astrocytes in striated nucleus: mean \pm SE of astrocytes in striated nucleus in 10 random areas at $40 \times$, each area $=35,500 \mu^{2}$. All this was carried out for the different treatment groups: control, experimental autoimmune encephalitis (EAE), and EAE + $N$-acetylcysteine (EAE + NAC). ${ }^{*} p<0.05$ vs control; ${ }^{*} p<0.05$ vs EAE

Rats with EAE showed clinical symptoms such as weight loss [37], edema and paralysis of the limbs, and tarnishing of the fur. Neurohistologic changes were also seen (Supplementary material S4 and S5). A decrease in neuronal density was indicative of cell loss through neuronal degeneration that causes the death of neurons by apoptosis and/or necrosis. These changes were corroborated by high levels of caspase- 3 and LDH in blood and brain; astrocytic proliferation

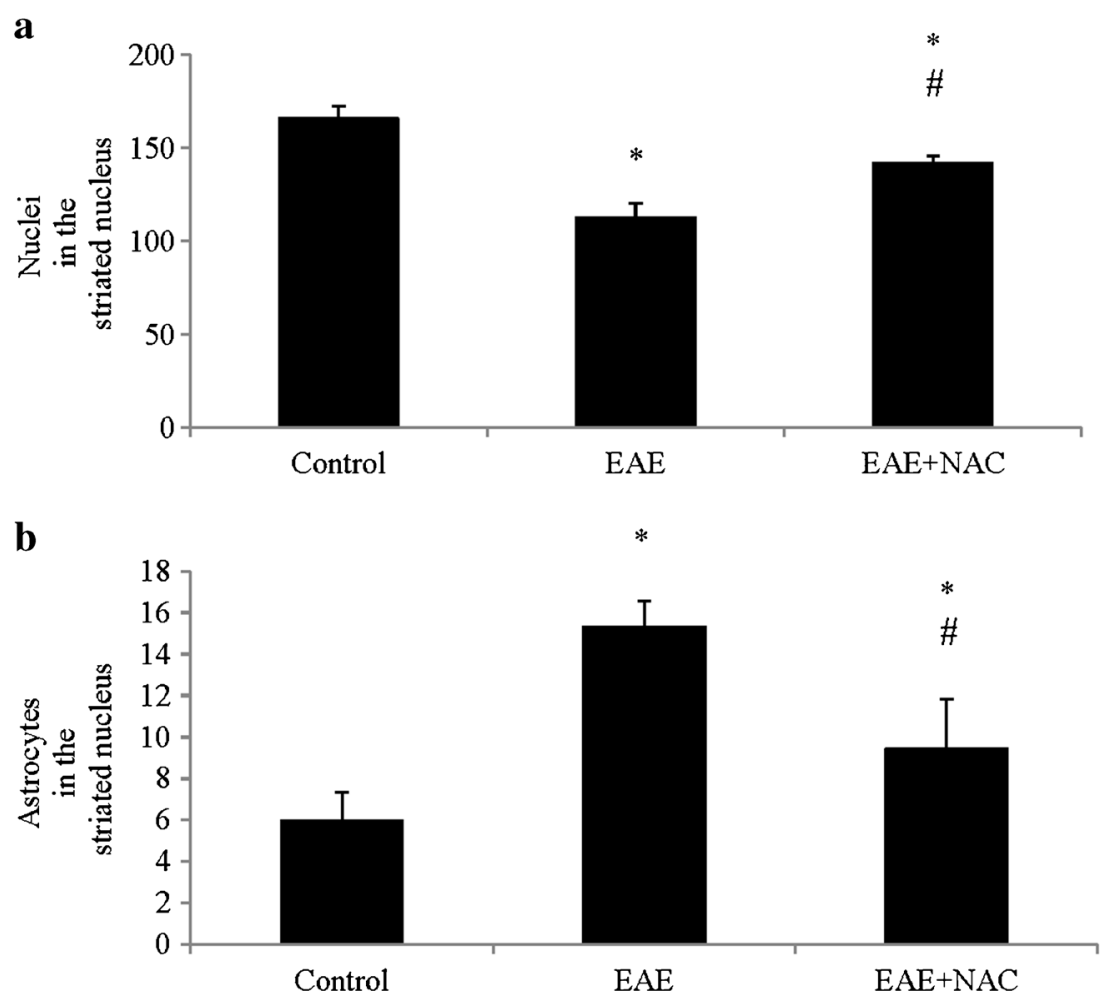

Fig. 6 Patients with relapsingremitting multiple sclerosis (RRMS): Mean \pm SE for the values of the bacterial lipopolysaccharide binding protein (LBP) in plasma $(\mu \mathrm{g} / \mathrm{ml})$ in 8 patients with RRMS ( 2 men, 6 women). MS-Baseline $=$ previous treatment with natalizumab; MS-56 = after 56 weeks of treatment with natalizumab. $* p<0.05$ vs control; $\bullet p<0.01$ vs MS-Baseline was also seen. Gliosis, especially astrogliosis, is characterized by astrocytic proliferation, extensive hypertrophy of the cell body, and functional changes, when stimulated with various factors, including LPS [17, 18].

The cause of MS is unknown, but it is known to be multifactorial; some studies suggest that oxidative stress may be one of the sources or a consequence of the disease from loss of oxidant/antioxidant balance $[25,38-40]$. ROS leading to

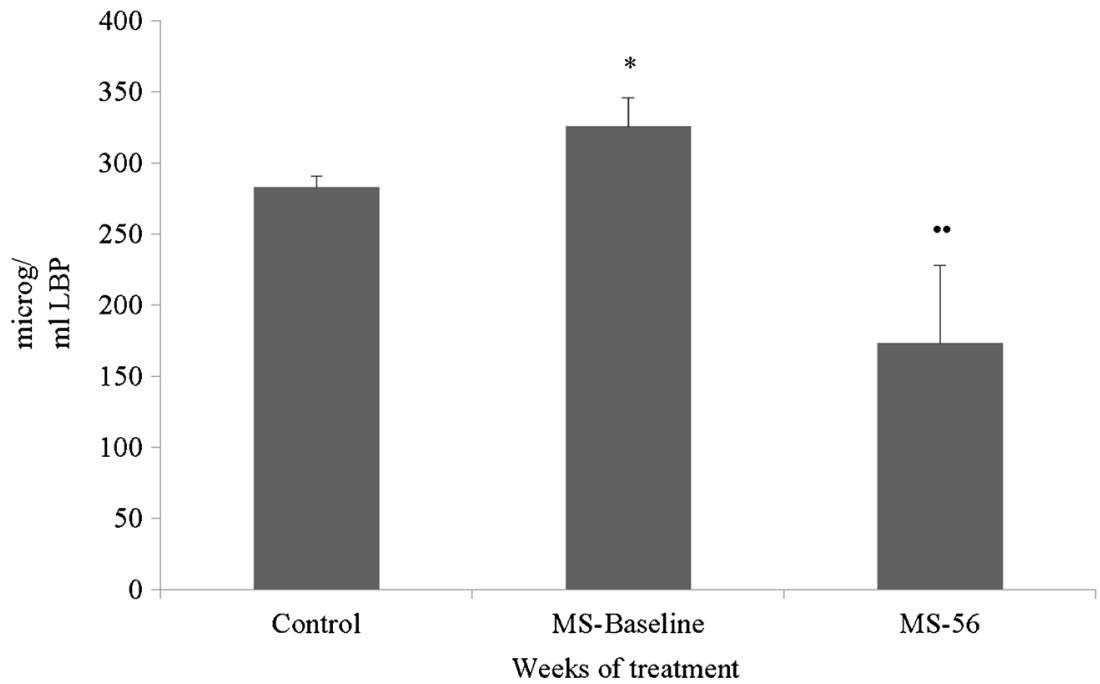


oxidative stress, generated in excess primarily by macrophages, have been implicated as mediators of demyelination and axonal damage in MS. Oxidative stress has even been linked to the course of the disease. Therefore, in patients with RRMS the increase in antioxidants supports the hypothesis that the response to oxidative damage in an attempt to protect cells is likely to occur before the inflammatory response, and, when this response is insufficient, a relapse in MS occurs [25]. It is well known that the CNS is particularly susceptible to ROS-induced damage on account of the high rate of oxygen utilization by the brain, the relatively poor concentrations of classic antioxidants, and the high content of polyunsaturated fatty acids [25].

In the EAA rats, high levels of LPO in the brain and spinal cord, and carbonylated proteins in blood were observed, and a positive correlation between oxidative stress and LBP was displayed. Further, these correlations appeared to be relevant to the clinical course at 35 days in rats. It has recently been suggested that LPS is able to increase the intracellular content of ROS through the activation of nicotinamide adenine dinucleotide phosphate oxidase. ROS, in turn, serve as second messengers to enhance gene expression induced by LPS through the receptor pathway [41]. As a consequence, cultured glial cells challenged with LPS are currently considered to be a valuable in vitro model for investigating the inflammatory processes of the brain.

Recently, the relationship between microbiota and MS has also been studied. Alterations in gut microbiota may lead to dysregulation of immune responses both in the gut and in distal effector immune sites such as the CNS [4]. A study of EAE mice has demonstrated increased intestinal permeability, overexpression of the tight junction zonulin, and alterations in intestinal morphology, together with an increased infiltration of proinflammatory Th1/Th17 cells and a reduced regulatory T-cell number in the gut lamina itself, Peyer's patches, and mesenteric lymph nodes [37]. These findings showed that circulating autoreactive $T$ cells play an essential role for the pathologic changes in intestinal wall morphology and barrier properties, and exhibit a disruption of intestinal homeostasis in EAE. The role of the commensal bacteria maintains the fine balance of immune homeostasis and the regulation of autoimmune processes in the periphery, and the modification of the bacterial populations of the gut has been seen to alter the clinical outcome of EAE in mice [4], and in people with RRMS [12, 13].

Bacterial endotoxin promotes the synthesis of the LBP, and forms an LPS-LBP complex that binds to CD14 [42]. Ochoa-Reparaz et al. [4] showed the capacity of the specific bacterial polysaccharide antigen to mediate trafficking and migration of a population of gut-derived antigen-presenting cells to CNS-associated lymphoid tissue. It is generally agreed that LPS exerts most of its pathologic effects by means of the activation of a specific receptor, named LBP/CD14/Toll-like receptor 4. Although early studies suggested that these receptors were specific to immunoinflammatory cells such as monocytes, later ones showed that they were also expressed in rat astrocytes [43, 44].

Our study showed that LPS and LBP increased in brain, spinal cord, and blood. This increase in LBP was reversed towards normality after treatment with natalizumab. Similar effects were seen in blood of patients with RRMS. In addition, NAC and DMF reduced levels of LBP in rat brain and spinal cord tissue. However, data not given in the present study show that LPS levels systemically increased by $118.31 \%$ in 2 patients with MS compared with the control group. Recently, various treatments for MS have been used in order to establish relationships in the MS-microbiotaoxidative damage axis.

Thus, in this work, the LPO levels in brain and spinal cord of EAE + NAC animals were reduced with respect to the EAE animal. NAC is a precursor of GSH, the main cellular antioxidant. NAC attenuates 4-hydroxy-2-nonenal-provoked redox changes in endothelial cells, prevents pathologic modification in tight junction protein network [45], and appears to prevent T-cell apoptosis and activity suppression induced by different agents, most likely by reducing the redox switches [46, 47]. Ljubisavljevic et al. [48] reported attenuated oxidative stress in the CNS of an EAE model, but in our study the LBP values were also reduced with the treatment.

The second of the treatments, DMF, has antioxidative/ neuroprotective and anti-inflammatory effects observed in vitro and in MS animal models [49]. In this study, caspase-3 in blood, as an indicator of apoptosis, decreased with treatment with DMF with respect to the EAE animal, and the LPO value also decreased with respect to EAE group in spinal cord. Experiments performed in astrocytes and neuronal cells with DMF showed that short-term exposure to fumarates results in a decreased intracellular GSH level and short-lived oxidative stress [27, 50-52]. However, long-term exposure showed the opposite effect, resulting in increased GSH levels and an activated antioxidative system [27, 50, 51]. Although progressive multifocal encephalopathy has been reported anecdotally in 5 of $>196,000$ patientyears of experience with fumaric acid esters, none of the 65,000 DMF-treated patients with MS treated in the first year were affected [53]. With regard to antiinflammatory effects of fumarates, it has been reported that DMF causes T-cell apoptosis both in vitro and in vivo (in a cuprizone model of MS) [27, 54, 55].

Finally, natalizumab is reported to be the most effective drug for MS, reducing both lesion formation [56] and 
axonal damage [57] in RRMS. Natalizumab is a monoclonal antibody directed against the $\alpha-4$ subunit of the $\alpha-4 /$ $\beta-1$ heterodimeric very late antigen-4 molecule, which is constitutively expressed on mononuclear immune cells. $\alpha-4-\beta-1-$ Integrin plays a major role in the transmigration of immune cells across the blood-brain barrier [58, 59]. Natalizumab is an effective therapy in RRMS as it reduces lymphocyte transmigration through the blood-brain barrier and induces lymphocytosis [60]. Natalizumab prompts a reduction in oxidative damage biomarker levels, together with a reduction both in myeloperoxidase levels and in the myeloperoxidase/neutrophil granulocyte ratio $[58,59]$. In this study, treatment with natalizumab decreased LPO levels in brain and spinal cord, and carbonylated proteins in blood with respect to the EAE group. This is produced because natalizumab suppresses the infiltration of pathogenic immune cells into the CNS [60], reducing oxidative stress. Additionally, as already mentioned, data not shown reveal that treatment with natalizumab for 56 weeks reduces LPS values by $64.29 \%$ in patients with RRMS compared with baseline values. MS drugs may reduce the role of commensal gut flora in triggering immune processes, leading to a RR autoimmune disease driven by myelin-specific $\mathrm{CD}^{+} \mathrm{T}$ cells [9].

Interestingly, 18 (5 men, 13 women) natalizumab-treated patients with RRMS showed an improvement in their general condition, characterized by a reduction in the number of relapses and recovery associated with major levels of melatonin in peripheral blood. These events were related to reduce oxidative damage and a decline in adhesion factors such as vascular cell adhesion molecule [21]. Also noteworthy is that natalizumab induced nuclear translocation of $\mathrm{Nrf2}$ and a fall in serum vascular cell adhesion molecule-1 levels in 22 patients with MS fulfilling the revised McDonald criteria, who were assigned to treatment with $300 \mathrm{mg}$ natalizumab intravenously once-monthly (infusion every 4 weeks) in accordance with Spanish guidelines [61].

In the light of these data, and in relation to those previously published by our group regarding patients with RRMS [62], it might be thought that changes in bacterial flora could cause the development of subacute inflammation. This process would entail the presence of systemic oxidative damage, which would start up the peripheral activation of the immune system with an alteration in the expression of vascular adhesion molecules and of the hematoencephalic barrier. All this would facilitate the transmigration of white cells to the CNS and the appearance of the neuroinflammatory phenomenon (Fig. 7).

It is of interest to note that this study could have a series of weaknesses such as the composition of the groups analyzed in each experiment, as well as the number of animals per group, or the impossibility of

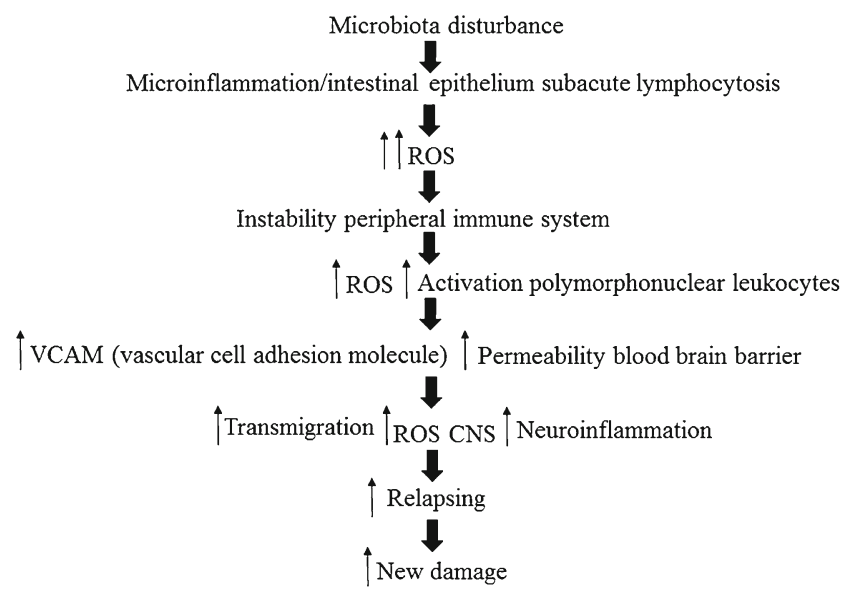

Fig. 7 Sketch of the possible cascade of events. ROS = reactive oxygen species; CNS = central nervous system

demonstrating the gut dysbiosis of the microbiota and the increased intestinal barrier permeability in the EAE rat. At the same time, it would be important to carry out some clinical studies to corroborate the same phenomena in patients with neuroinflammatory processes, especially those with MS, in order to enable us to find out the biochemical mechanisms involved in these processes and to design new therapeutic strategies with a greater clarity.

In summary, it can be concluded that these data once again back up the important role played by oxidative stress in the establishment, course. and evolution of neuroinflammatory processes like MS, and in those experimentally induced like EAE. The changes of LBP in EAE, and possibly in MS, imply the important role played by LPS and its close relationship in the homeostasis and hormesis of the bacterial flora-immune system-CNS axis. There is a possibility of designing new therapeutic targets using our data for the treatment of neuroinflammatory processes like MS. Finally, more studies are necessary to further understand the mechanisms involved and their interactions between LBP and LPS, inflammation, and oxidative stress.

Acknowledgments We thank M. La Torre for technical support in the collection and laboratory analyses of the samples.

Required Author Forms Disclosure forms provided by the authors are available with the online version of this article.

\section{References}

1. McQualter JL, Bernard CC. Multiple sclerosis: a battle between destruction and repair. J Neurochem 2007;100:295-306.

2. Perez-Nievas BG, Garcia-Bueno B, Madrigal JL, Leza JC. Chronic immobilisation stress ameliorates clinical score and 
neuroinflammation in a MOG-induced EAE in Dark Agouti rats: mechanisms implicated. J Neuroinflammation 2010;7:60.

3. Lucchinetti C, Bruck W, Parisi J, Scheithauer B, Rodriguez M, Lassmann H. Heterogeneity of multiple sclerosis lesions: implications for the pathogenesis of demyelination. Ann Neurol 2000;47: 707-717.

4. Ochoa-Reparaz J, Mielcarz DW, Begum-Haque S, Kasper LH. Gut, bugs, and brain: role of commensal bacteria in the control of central nervous system disease. Ann Neurol 2011;69:240-247.

5. Wang Y, Kasper LH. The role of microbiome in central nervous system disorders. Brain Behav Immun 2014;38:1-12.

6. Kabat EA, Wolf A, Bezer AE. Rapid production of acute disseminated encephalomyelitis in Rhesus monkeys by injection of brain tissue with adjuvants. Science 1946;104:362-363.

7. Clemente JC, Ursell LK, Parfrey LW, Knight R. The impact of the gut microbiota on human health: an integrative view. Cell 2012: 148:1258-1270.

8. Yokote H, Miyake S, Croxford JL, Oki S, Mizusawa H, Yamamura T. NKT cell-dependent amelioration of a mouse model of multiple sclerosis by altering gut flora. Am J Pathol 2008:173:1714-1723.

9. Berer K, Mues M, Koutroulos M, et al. Commensal microbiota and myelin autoantigen cooperate to trigger autoimmune demyelination. Nature 2011:479:538-541.

10. Legroux L, Arbour N. Multiple sclerosis and T lymphocytes: an entangled story (review). J Neuroimmune Pharmacol 2015:10:528546.

11. Wekerle H, Berer K, Krishnamoorthy G. Remote control-triggering of brain autoimmune disease in the gut. Curr Opin Immunol 2013;25:683-689.

12. Chen J, Chia N, Kalari KR, et al. Multiple sclerosis patients have a distinct gut microbiota compared to healthy controls. Sci Rep2016: 6:28484.

13. Jangi S, Gandhi R, Cox LM, et al. Alterations of the human gut microbiome in multiple sclerosis. Nat Commun 2016:7:12015.

14. Dasgupta S, Kasper DL. Novel tools for modulating immune responses in the host-polysaccharides from the capsule of commensal bacteria. Adv Immunol 2010;106:61-91.

15. Mazmanian SK, Kasper DL. The love-hate relationship between bacterial polysaccharides and the host immune system. Nat Rev Immunol 2006;6:849-858.

16. Riccio P. The molecular basis of nutritional intervention in multiple sclerosis: a narrative review. Complement Ther Med 2011;19:228-237.

17. Cani PD, Delzenne NM. The role of the gut microbiota in energy metabolism and metabolic disease. Curr Pharm Des 2009;15:15461558 .

18. Guarner F. Hygiene, microbial diversity and immune regulation. Curr Opin Gastroenterol 2007;23:667-672.

19. Veldhoen M, Hirota K, Westendorf AM, et al. The aryl hydrocarbon receptor links TH17-cell-mediated autoimmunity to environmental toxins. Nature 2008;453:106-109.

20. Kim SY, Lee JG, Cho WS, et al. Role of NADPH oxidase-2 in lipopolysaccharide-induced matrix metalloproteinase expression and cell migration. Immunol Cell Biol 2010:88:197-204.

21. Bahamonde C, Conde C, Aguera E, et al. Elevated melatonin levels in natalizumab-treated female patients with relapsing-remitting multiple sclerosis: relationship to oxidative stress. Eur J Pharmacol 2014;730:26-30

22. Melamud L, Golan D, Luboshitzky R, Lavi I, Miller A. Melatonin dysregulation, sleep disturbances and fatigue in multiple sclerosis. J Neurol Sci 2012;314:37-40.

23. Miller E, Walczak A, Saluk J, Ponczek MB, Majsterek I. Oxidative modification of patient's plasma proteins and its role in pathogenesis of multiple sclerosis. Clin Biochem 2012;45:26-30.

24. Tasset I, Aguera E, Gascon F, et al. Natalizumab and reduction of carbonylated proteins in patients with multiple sclerosis. Rev Neurol 2012;54:449-452.
25. Tasset I, Aguera E, Sanchez-Lopez F, et al. Peripheral oxidative stress in relapsing-remitting multiple sclerosis. Clin Biochem 2012;45:440-444.

26. Neish AS. Redox signaling mediated by the gut microbiota. Free Radic Res 2013;47:950-957.

27. Miljkovic D, Spasojevic I. Multiple sclerosis: molecular mechanisms and therapeutic opportunities. Antioxidant Redox Signal 2013;19:2286-2334.

28. Stosic-Grujicic S, Ramic Z, Bumbasirevic V, Harhaji L, MostaricaStojkovic M. Induction of experimental autoimmune encephalomyelitis in Dark Agouti rats without adjuvant. Clin Exp Immunol 2004;136:49-55

29. Deloire MS, Touil T, Brochet B, Dousset V, Caille JM, Petry KG. Macrophage brain infiltration in experimental autoimmune encephalomyelitis is not completely compromised by suppressed T-cell invasion: in vivo magnetic resonance imaging illustration in effective anti-VLA-4 antibody treatment. Mult Scler 2004;10:540-548.

30. Papadopoulos D, Rundle J, Patel R, et al. FTY720 ameliorates MOG-induced experimental autoimmune encephalomyelitis by suppressing both cellular and humoral immune responses. J Neurosci Res 2010;88:346-359.

31. Kamboj SS, Sandhir R. Protective effect of N-acetylcysteine supplementation on mitochondrial oxidative stress and mitochondrial enzymes in cerebral cortex of streptozotocin-treated diabetic rats. Mitochondrion 2011;11:214-222.

32. Milenkovic M, Arsenovic-Ranin N, Vucicevic D, Bufan B, Jancic I, Stojic-Vukanic Z. Beneficial effects of dimethyl fumarate on experimental autoimmune myocarditis. Arch Med Res 2008;39:639-646.

33. Polman CH, Wolinsky JS, Reingold SC. Multiple sclerosis diagnostic criteria: three years later. Mult Scler 2005;11:5-12.

34. Kurtzke JF. Rating neurologic impairment in multiple sclerosis: an expanded disability status scale (EDSS). Neurology 1983;33:14441452.

35. Levine RL, Garland D, Oliver CN, Amici A, Climent I, Lenz AG. Determination of carbonyl content in oxidatively modified proteins. Methods Enzymol 1990;186:464-478.

36. Trooster WJ, Teelken AW, Gerrits PO, et al. The effect of gonadectomy on the clinical course of chronic experimental allergic encephalomyelitis. Clin Neurol Neurosurg 1996;98:222-226.

37. Nouri M, Bredberg A, Westrom B, Lavasani S. Intestinal barrier dysfunction develops at the onset of experimental autoimmune encephalomyelitis, and can be induced by adoptive transfer of autoreactive T cells. PLOS ONE 2014;9:e106335.

38. Calabrese V, Bella R, Testa D, et al. Increased cerebrospinal fluid and plasma levels of ultraweak chemiluminescence are associated with changes in the thiol pool and lipid-soluble fluorescence in multiple sclerosis: the pathogenic role of oxidative stress. Drugs Exp Clin Res 1998;24:125-131.

39. Ortiz GG, Macias-Islas MA, Pacheco-Moises FP, et al. Oxidative stress is increased in serum from Mexican patients with relapsingremitting multiple sclerosis. Disease Markers 2009:26:35-39.

40. Ferreira B, Mendes F, Osorio N, Caseiro A, Gabriel A, Valado A. Glutathione in multiple sclerosis. Br J Biomed Sci 2013;70:75-79.

41. Wang T, Qin L, Liu B, et al. Role of reactive oxygen species in LPSinduced production of prostaglandin E2 in microglia. J Neurochem 2004;88:939-947.

42. Bannerman DD, Paape MJ, Hare WR, Sohn EJ. Increased levels of LPS-binding protein in bovine blood and milk following bacterial lipopolysaccharide challenge. J Dairy Sci 2003;86:3128-3137.

43. Galea E, Reis DJ, Fox ES, Xu H, Feinstein DL. CD14 mediate endotoxin induction of nitric oxide synthase in cultured brain glial cells. J Neuroimmunol 1996;64:19-28.

44. Mancuso C, Scapagini G, Curro D, et al. Mitochondrial dysfunction, free radical generation and cellular stress response in neurodegenerative disorders. Front Biosci 2007;12:1107-1123. 
45. Usatyuk PV, Parinandi NL, Natarajan V. Redox regulation of 4hydroxy-2-nonenal-mediated endothelial barrier dysfunction by focal adhesion, adherens, and tight junction proteins. J Biol Chem 2006;281:35554-35566.

46. Breithaupt TB, Vazquez A, Baez I, Eylar EH. The suppression of $\mathrm{T}$ cell function and $\mathrm{NF}(\mathrm{kappa}) \mathrm{B}$ expression by serine protease inhibitors is blocked by $\mathrm{N}$-acetylcysteine. Cell Immunol 1996;173:124-130.

47. Heussler VT, Fernandez PC, Machado J, Jr, Botteron C, Dobbelaere DA. N-acetylcysteine blocks apoptosis induced by $\mathrm{N}$-alpha-tosylL-phenylalanine chloromethyl ketone in transformed T-cells. Cell Death Differ 1999;6:342-350.

48. Ljubisavljevic S, Stojanovic I, Pavlovic D, Sokolovic D, Stevanovic I. Aminoguanidine and N-acetyl-cysteine supress oxidative and nitrosative stress in EAE rat brains. Redox Rep 2011;16:166-172.

49. Kappos L, Gold R, Miller DH, et al. Efficacy and safety of oral fumarate in patients with relapsing-remitting multiple sclerosis: a multicentre, randomised, double-blind, placebo-controlled phase IIb study. Lancet 2008;372:1463-1472.

50. Albrecht P, Bouchachia I, Goebels N, et al. Effects of dimethyl fumarate on neuroprotection and immunomodulation. J Neuroinflammation 2012;9:163.

51. Lin SX, Lisi L, Dello Russo C, et al. The anti-inflammatory effects of dimethyl fumarate in astrocytes involve glutathione and haem oxygenase-1. ASN Neuro 2011;3.

52. Schmidt MM, Dringen R. Fumaric acid diesters deprive cultured primary astrocytes rapidly of glutathione. Neurochem Int 2010;57: 460-467.
53. Sheremata W, Brown AD, Rammohan KW. Dimethyl fumarate for treating relapsing multiple sclerosis. Exp Opin Drug Saf 2015;14:161-170.

54. Moharregh-Khiabani D, Blank A, Skripuletz T, et al. Effects of fumaric acids on cuprizone induced central nervous system deand remyelination in the mouse. PLOS ONE 2010;5:e11769.

55. Treumer F, Zhu K, Glaser R, Mrowietz U. Dimethylfumarate is a potent inducer of apoptosis in human T cells. J Invest Dermatol 2003; 121:1383-1388.

56. Radue EW, Stuart WH, Calabresi PA, et al. Natalizumab plus interferon beta-1a reduces lesion formation in relapsing multiple sclerosis. J Neurol Sci 2010;292:28-35.

57. Gunnarsson M, Malmestrom C, Axelsson M, et al. Axonal damage in relapsing multiple sclerosis is markedly reduced by natalizumab. Ann Neurol 2011;69:83-89.

58. Bielekova B, Becker BL. Monoclonal antibodies in MS: mechanisms of action. Neurology 2010;74(Suppl. 1):S31-S40.

59. Trebst C, Voss E, Skripuletz T, Stangel M. Specific immune intervention with monoclonal antibodies for the treatment of multiple sclerosis. Curr Med Chem 2010;17:640-650.

60. Signoriello E, Lanzillo R, Brescia Morra V, et al. Lymphocytosis as a response biomarker of natalizumab therapeutic efficacy in multiple sclerosis. Mult Scler 2016:22:921-925.

61. Tasset I, Bahamonde C, Aguera E, et al. Effect of natalizumab on oxidative damage biomarkers in relapsing-remitting multiple sclerosis. Pharmacol Rep 2013;65:624-631.

62. Escribano BM, Túnez I. Gut microbiota and central nervous system condemned to understand each other: their role in multiple sclerosis. MOJ Cell Sci Rep 2014;1:00005. 